\title{
Rate-Ratio Asymptotic Analysis of the Influence of Stoichiometric Mixture Fraction on Structure and Extinction of Laminar, Nonpremixed Methane Flames with Comparison to Experiments
}

Philipp Mairhofer ${ }^{(a, b)}$, Gerald Mairinger ${ }^{(a, b)}$, Kalyanasundaram Seshadri ${ }^{(a)}$, Xue-Song Bai ${ }^{(c)}$, Reinhard Seiser ${ }^{(a)}$, Ernst Pucher ${ }^{(b)}$,

(a) Department of Mechanical and Aerospace Engineering, University of California at San Diego, La Jolla, California 92093-0411, USA,

${ }^{(b)}$ Institute for Powertrains and Automotive Technology, Vienna University of Technology, A-1040 Vienna, Gusshausstrasse 27-29/315, Austria,

(c) Division of Fluid Mechanics, Department of Energy Sciences, Lund University, S 22100 Lund, Sweden.

Corresponding author:

K. Seshadri,

Department of Mechanical and Aerospace Engineering,

University of California at San Diego,

La Jolla, California 92093-0411,

Phone: (858) 534-4876. Fax: (858) 534-5354,

email: seshadri@ucsd.edu

- Revised manuscript \# PROCI-D-15-00870

- Preferred colloquium topic: Laminar Flames.

- Word-Length: 5850 words

- Method 2 was used. The paper was formatted in the two column format recommended by Elsevier (documentclass[final,5p,times,twocolumn]\{elsarticle\}). The length of the paper (excluding abstract, title block and separate list of figure captions) was 6.5 pages.

- Total number of words $6.5 \times 900=5850$ words

- The color reproduction charges will be paid if applicable

Thirty-Sixth International Symposium on Combustion

COEX, Seoul, Korea

July 31-August 5, 2016 


\title{
Rate-Ratio Asymptotic Analysis of the Influence of Stoichiometric Mixture Fraction on Structure and Extinction of Laminar, Nonpremixed Methane Flames with Comparison to Experiments
}

\author{
Philipp Mairhofer ${ }^{\mathrm{a}, \mathrm{b}}$, Gerald Mairinger ${ }^{\mathrm{a}, \mathrm{b}}$, Kalyanasundaram Seshadri ${ }^{\mathrm{a}}$, \\ Xue-Song Baic ${ }^{\mathrm{c}}$, Reinhard Seiser ${ }^{\mathrm{a}}$, Ernst Pucher ${ }^{\mathrm{b}}$ \\ ${ }^{a}$ Department of Mechanical and Aerospace Engineering, University of California at San \\ Diego, La Jolla, California 92093-0411, USA \\ ${ }^{b}$ Institute for Powertrains and Automotive Technology, Vienna University of Technology, \\ A-1040 Vienna, Gusshausstrasse 27-29/315, Austria \\ ${ }^{c}$ Division of Fluid Mechanics, Department of Energy Sciences, Lund University, 22100 \\ Lund, Sweden
}

\begin{abstract}
Activation energy asymptotic analysis and rate-ratio asymptotic analysis of combustion in laminar, nonpremixed flames are often carried out using conserved scalar quantities as independent variables. One such representation of a conserved scalar quantity is the mixture fraction, $\xi$, based on thermal diffusivity. These analyses are carried out in the asymptotic limit of large Damköhler number, with chemical reactions presumed to take place in a thin reaction zone that is located at $\xi=\xi_{\text {st }}$. The quantity $\xi_{\text {st }}$ is the stoichiometric mixture fraction. A characteristic diffusion time is given by the reciprocal of the scalar dissipation rate, $\chi$. Previous computational studies have shown that the scalar dissipation rate at extinction depends on $\xi_{\text {st }}$ and the maximum flame temperature, $T_{\mathrm{st}}$. Here, a rate-ratio asymptotic analysis is carried out using reduced chemistry to elucidate the influence of $\xi_{\text {st }}$ on critical conditions
\end{abstract}


of extinction. The scalar dissipation rate at extinction was predicted as a function of $\xi_{\text {st }}$ with the mass fractions of reactants so chosen that the adiabatic flame temperature, $T_{\mathrm{st}}$, is fixed. The predictions of the analysis show that with increasing values of $\xi_{\text {st }}$, the scalar dissipation rate at extinction first increases and then decreases. To test the predictions of the asymptotic analysis, critical conditions of extinction are measured on nonpremixed methane flames stabilized in the counterflow configuration. With increasing values of stoichiometric mixture fraction, the strain rate at extinction was found to increase, and the scalar dissipation rate at extinction was found to first increase and then decrease. The predictions of the asymptotic analysis agreed with experiments. A key outcome of the analysis is that with increasing $\xi_{\text {st }}$, the thickness of the regions where oxygen and fuel are consumed first increase and the decrease. This is responsible for the observed non-monotonic changes in the values of the scalar dissipation rate at extinction with changes in $\xi_{\text {st }}$.

Keywords: rate-ratio asymptotic analysis, conserved scalar, methane flames, extinction, flame structure

\section{Introduction}

Conserved scalar quantities are frequently employed in fundamental studies of the structure of laminar, nonpremixed flames [1-6]. Nonpremixed combustion takes place in mixing layers between a fuel stream that contains the fuel, and an oxidizer stream that contains oxygen. In analytical studies it is convenient to use these conserved scalar quantities as independent variables. There are many approaches for constructing these variables and they 
differ in the manner by which the diffusion of the conserved scalar is characterized [7-9]. One such construction is to require the conserved scalar quantity represented by $\xi$, to satisfy the source-free conservation equation, $\rho(\vec{v} \cdot \vec{\nabla}) \xi-\vec{\nabla} \cdot\left[\left(\lambda / c_{\mathrm{p}}\right) \vec{\nabla} \xi\right]=0$, where $\vec{v}$ is the velocity vector, $\rho$ the density, $\lambda$ the thermal conductivity, and $c_{\mathrm{p}}$ the heat capacity per unit mass of the mixture. This equation for $\xi$ is constrained to satisfy the conditions, $\xi=0$ in the oxidizer stream far from the mixing layer and $\xi=1$ in the fuel stream [1-6]. A characteristic diffusion is given by the reciprocal of the scalar dissipation rate, $\chi=2\left[\lambda /\left(\rho c_{\mathrm{p}}\right)\right]|\vec{\nabla} \xi|^{2},[1-6]$.

In asymptotic analysis of flame structure, all chemical reactions are presumed to take place in a thin reaction zone, that is located at $\xi=\xi_{\text {st }}$. To the leading order, the mass fluxes of fuel and oxygen into the reaction zone are in stoichiometric proportions. On either side of this reaction zone, the chemical reactions are presumed to be frozen [1-5] or the flow-field is inert $[6,9-12]$. The scalar dissipation rate at $\xi=\xi_{\text {st }}$ is represented by $\chi_{\text {st }}$. It has been established from activation-energy asymptotic analysis (AEA) [1-5] and rate-ratio asymptotic analysis (RRA) [6, 9-12] that the flame structure and critical conditions of extinction depend on $\xi_{\text {st }}$ and $T_{\text {st }}$, where $T_{\text {st }}$ is the adiabatic flame temperature.

Grudno and Seshadri [13] have carried out a detailed computational study of the influences of $\xi_{\mathrm{st}}$ and $T_{\mathrm{st}}$ on the strain rate at extinction, $a_{\mathrm{q}}$, and scalar dissipation rate at extinction, $\chi_{\text {st,q }}$, where the subscript $q$ represents conditions at extinction. This study was carried out for $\xi_{\text {st }}<0.5$. The results 
show that at fixed $\xi_{\mathrm{st}}$, the value of $\chi_{\mathrm{st}, \mathrm{q}}$ and $a_{\mathrm{q}}$ increase with increasing values of $T_{\mathrm{st}}$. At fixed $T_{\mathrm{st}}, \chi_{\mathrm{st}, \mathrm{q}}$ and $a_{\mathrm{q}}$ also increase with increasing $\xi_{\mathrm{st}}$. Chen and Axelbaum [14] have measured critical conditions of extinction for methane flames at fixed $T_{\text {st }}$, and their results show that the strain rate at extinction increases with increasing $\xi_{\text {st. }}$. Song et al. [15] carried out an experimental and computational investigation of the effects of $\xi_{\text {st }}$ on propagation speeds of edge flames. They found a close correlation between the influence of $\xi_{\text {st }}$ on edge flame propagation speed and the influence of $\xi_{\text {st }}$ on critical conditions of extinction of nonpremixed flames. In the present study, a rate-ratio asymptotic analysis is carried out to obtain an improved understanding of the influence of $\xi_{\text {st }}$ on flame structure and critical conditions of extinction. Although, the fuel considered here is methane, the results of the analysis can be applied to other hydrocarbon fuels. Critical conditions of extinction are predicted. To test the validity of the analysis experiments are carried out on counterflow methane flame. Critical conditions of extinction are measured at fixed values of $T_{\mathrm{st}}$ and various values of $\xi_{\mathrm{st}}$. The predictions of the RRA analysis are compared with the measurements.

\section{Rate-Ratio Asymptotic Analysis}

Previous rate-ratio asymptotic analyses of methane flames were applied to problems where $\xi_{\text {st }}$ was small $[9,12]$. Here, the analysis is extended to include values of $\xi_{\text {st }}>0.5$. Consider a steady nonpremixed methane flame stabilized in the mixing layer between two laminar streams. One stream, called the fuel stream, is a mixture of methane and nitrogen, and the other stream, called the oxidizer stream, is a mixture of oxygen and nitrogen. In the fuel stream 
far from the mixing layer, the mass fraction of fuel is represented by $Y_{\mathrm{F}, 1}$ and the temperature by $T_{1}$, and in the oxidizer stream, far from the mixing layer the mass fraction of oxygen is represented by $Y_{\mathrm{O}_{2}, 2}$, and the temperature by $T_{2}$. Here, subscript $\mathrm{F}$ refers to methane, and subscripts 1 and 2, respectively, refer to conditions in the fuel stream and oxidizer stream. If the diffusivity of methane, oxygen, and nitrogen are presumed to be equal to the thermal diffusivity, it has been shown that [9] the stoichiometric mixture fraction, $\xi_{\text {st }}$, is given by

$$
\xi_{\text {st }}=\left[1+2 Y_{\mathrm{F}, 1} W_{\mathrm{O}_{2}} /\left(Y_{\mathrm{O}_{2}, 2} W_{\mathrm{F}}\right)\right]^{-1}
$$

and the adiabatic temperature by

$$
T_{\mathrm{st}}=T_{\mathrm{u}}+Q_{\mathrm{F}} \xi_{\mathrm{st}} Y_{\mathrm{F}, 1} /\left(W_{\mathrm{F}} c_{\mathrm{p}, \mathrm{st}}\right)
$$

where $W_{\mathrm{F}}$ and $W_{\mathrm{O}_{2}}$ are the molecular weights of methane and oxygen, $T_{\mathrm{u}}=$ $T_{2}+\xi_{\text {st }}\left(T_{1}-T_{2}\right), Q_{\mathrm{F}}$ is the heat release in the reaction $\mathrm{CH}_{4}+2 \mathrm{O}_{2} \rightarrow \mathrm{CO}_{2}$ $+2 \mathrm{H}_{2} \mathrm{O}$, and $c_{\mathrm{p}, \mathrm{st}}$ is the heat capacity per unit mass of the products.

For convenience, the definitions

$$
X_{\mathrm{i}} \equiv Y_{\mathrm{i}} W_{\mathrm{N}_{2}} / W_{\mathrm{i}}, \quad \tau \equiv c_{\mathrm{p}} W_{\mathrm{N}_{2}}\left(T-T_{\mathrm{u}}\right) / Q_{\mathrm{F}},
$$

are introduced. Here $Y_{\mathrm{i}}$, and $W_{\mathrm{i}}$ are respectively the mass fraction and molecular weight of species $i$ and $W_{\mathrm{N}_{2}}$ is the molecular weight of nitrogen. In the analysis, the approximation that the value of the Lewis number of species $i, L e_{\mathrm{i}}=\lambda /\left(\rho c_{\mathrm{p}} D_{\mathrm{i}}\right)$, is unity for $\mathrm{CH}_{4}, \mathrm{O}_{2}, \mathrm{CO}_{2}, \mathrm{H}_{2} \mathrm{O}$, and $\mathrm{N}_{2}$ is introduced, where $D_{\mathrm{i}}$ is the coefficient of diffusion of species $i$. The conserved scalar quantity, $\xi$, is used as the independent variable. As a consequence, the 
profile of temperature and profiles of mass fractions of all species are evaluated as a function of $\xi$. Chemical reactions are presumed to take place in a thin reaction-zone that is located at $\xi_{\text {st }}$. The regions $0 \leq \xi<\xi_{\text {st }}$, and $\xi_{\text {st }}<\xi \leq 1.0$, are inert and represent the outer structure. Analysis of the outer structure provides matching conditions for the equations describing the structure of the reaction zone.

\subsection{Outer Structure}

The profile of temperature, $T$, and profiles of mass fractions of the reactants $\mathrm{CH}_{4}$, and $\mathrm{O}_{2}$, and the products $\mathrm{CO}_{2}$, and $\mathrm{H}_{2} \mathrm{O}$ represent the outer structure of the flame. These profiles are linear functions of $\xi$, and for constant $c_{\mathrm{p}}$, the profile of temperature $T$ is also a linear function of $\xi$. At the reaction zone located at $\xi=\xi_{\text {st }}$, the mass fractions of $\mathrm{CH}_{4}$, and $\mathrm{O}_{2}$, are zero. In the region $\xi>\xi_{\mathrm{st}}, Y_{\mathrm{O}_{2}}=0$, and in the region $\xi<\xi_{\mathrm{st}}, Y_{\mathrm{F}}=0$. The gradient of $\tau$, and those of $X_{\mathrm{i}}$ with respect to $\xi$, are discontinuous at $\xi=\xi_{\text {st }}$. These gradients in the region $\xi>\xi_{\text {st }}$, represented by the subscript + , are $\left(d X_{\mathrm{F}} / d \xi\right)_{+}=X_{\mathrm{F}, 1} /\left(1-\xi_{\mathrm{st}}\right)=g,(d \tau / d \xi)_{+}=-\tau_{\mathrm{st}} /\left(1-\xi_{\mathrm{st}}\right)=-p$, and in the region $\xi<\xi_{\text {st }}$, represented by the subscript - , are $\left(d X_{\mathrm{O}_{2}} / d \xi\right)_{-}=$ $-X_{\mathrm{O}_{2}, 2} / \xi_{\mathrm{st}}=-2 g,(d \tau / d \xi)_{-}=\tau_{\mathrm{st}} / \xi_{\mathrm{st}}=s$. Element balance gives the relations $X_{\mathrm{CO}_{2}, \mathrm{st}}=g \xi_{\mathrm{st}}\left(1-\xi_{\mathrm{st}}\right)$, and $X_{\mathrm{H}_{2} \mathrm{O}, \mathrm{st}}=2 g \xi_{\mathrm{st}}\left(1-\xi_{\mathrm{st}}\right)[9]$. It follows from Eqs. (2) and (3) that for constant values of the heat capacity $X_{\mathrm{F}, 1} \xi_{\mathrm{st}}=\tau_{\mathrm{st}}$,

hence $g=\tau_{\mathrm{st}}\left[\xi_{\mathrm{st}}\left(1-\xi_{\mathrm{st}}\right)\right]^{-1}$. As a consequence $g$ is proportional to the sum of the absolute values of the gradient of temperature with respect to $\xi$ in the outer structure. 


\subsection{Reduced Mechanism}

The reduced four-step mechanism that is employed to describe the chemical reactions taking place in the thin reaction zone at $\xi=\xi_{\text {st }}$ is (I) $\mathrm{CH}_{4}+$ $2 \mathrm{H}+\mathrm{H}_{2} \mathrm{O} \rightleftharpoons \mathrm{CO}+4 \mathrm{H}_{2},\left(\right.$ II) $\mathrm{CO}+\mathrm{H}_{2} \mathrm{O} \rightleftharpoons \mathrm{CO}_{2}+\mathrm{H}_{2}$, (III) $\mathrm{H}+\mathrm{H}+\mathrm{M} \rightarrow$ $\mathrm{H}_{2}+\mathrm{M}$, and (IV) $\mathrm{O}_{2}+3 \mathrm{H}_{2} \rightleftharpoons 2 \mathrm{H}+2 \mathrm{H}_{2} \mathrm{O}$. Here, global step I represents reactions between fuel and radicals to form the intermediate product $\mathrm{CO}$ and $\mathrm{H}_{2}$. The global step II represents formation of $\mathrm{CO}_{2}$ from $\mathrm{CO}$, the global step III represents chain-breaking reactions and global step IV chain-branching reactions. Table 1 shows the elementary reactions that contribute to the

Table 1: Rate data. Units are moles, cubic centimeters, seconds, kJoules, Kelvin.

\begin{tabular}{|c|c|c|c|c|c|c|}
\hline Number & \multicolumn{3}{|c|}{ Reaction } & $B_{n}$ & $\alpha_{n}$ & $E_{n}$ \\
\hline $1 \mathrm{f}$ & $\mathrm{O}_{2}+\mathrm{H}$ & $\rightarrow$ & $\mathrm{OH}+\mathrm{O}$ & $2.000 \times 10^{14}$ & 0.00 & 70.30 \\
\hline 5 & $\mathrm{H}+\mathrm{O}_{2}+\mathrm{M}$ & $\rightarrow$ & $\mathrm{HO}_{2}+\mathrm{M}$ & $2.300 \times 10^{18}$ & -0.80 & 0.00 \\
\hline $6 f$ & $\mathrm{CO}+\mathrm{OH}$ & $\rightarrow$ & $\mathrm{CO}_{2}+\mathrm{H}$ & $4.400 \times 10^{6}$ & 1.50 & -3.10 \\
\hline $7 \mathrm{f}$ & $\mathrm{CH}_{4}+\mathrm{H}$ & $\rightarrow$ & $\mathrm{CH}_{3}+\mathrm{H}_{2}$ & $2.200 \times 10^{4}$ & 3.00 & 36.60 \\
\hline \multirow[t]{2}{*}{8} & \multirow[t]{2}{*}{$\mathrm{CH}_{3}+\mathrm{H}$} & \multirow{2}{*}{\multicolumn{2}{|c|}{$\rightarrow \quad \mathrm{CH}_{4}$}} & $6.257 \times 10^{23}$ & -1.80 & 0.00 \\
\hline & & & & $2.108 \times 10^{14}$ & 0.00 & 0.00 \\
\hline 9 & $\mathrm{CH}_{3}+\mathrm{O}$ & $\rightarrow$ & $\mathrm{CH}_{2} \mathrm{O}+\mathrm{H}$ & $7.000 \times 10^{13}$ & 0.00 & 0.00 \\
\hline
\end{tabular}

rate of these global steps of the reduced mechanism. They are the same as those employed in previous studies $[9,12]$. The symbol $f$ appearing in the first column of Table 1 refers to the forward step of a reversible elementary reaction $n$, and subscript $b$ later will refer to the reverse step. Reactions 5,8 , and 9 , are presumed to be irreversible. The rate constant of elementary step 
$n$ is $k_{n}=B_{n} T^{\alpha_{n}} \exp \left[-E_{n} /(\widehat{R} T)\right]$, where $\widehat{R}$ is the universal gas constant. The equilibrium constants for the reversible reactions 1, 6, and 7, respectively, are $K_{1}=12.76 \times \exp (-8032 / T), K_{6}=8.88 \times 10^{-3} \times \exp (11169 / T)$, and $K_{7}=26.21 \times \exp (-245 / T)$. The concentration of the third body is represented by $C_{\mathrm{M}}$. The rate constant for reaction 8 is calculated using the formula given in [16], and [12]. The elementary steps (2) $\mathrm{H}_{2}+\mathrm{O} \rightleftharpoons \mathrm{OH}+\mathrm{H}$, and (3) $\mathrm{H}_{2}+\mathrm{OH} \rightleftharpoons \mathrm{H}_{2} \mathrm{O}+\mathrm{H}$ are presumed to maintain partial equilibrium. The quantities $K_{2}=2.28 \times \exp (-963 / T)$, and $K_{3}=0.232 \times \exp (7536 / T)$ are, respectively, the equilibrium constant of the elementary steps (2) and (3).

\subsection{The Structure of the Reaction Zone}

The global reactions of the reduced four-step mechanism take place in various layers within the reaction zone [9]. Since radicals participate in all elementary steps, global step IV is active in the entire reaction zone. Figure 1 is a schematic illustration of the structure of the reaction zone. The profile of temperature and profiles of $\mathrm{CH}_{4}, \mathrm{O}_{2}, \mathrm{H}_{2}, \mathrm{CO}$, and $\mathrm{H}$ are shown. The temperature profiles shown by the dashed lines in Fig. 1 are projections from the outer structure into the reaction zone. The quantity $T^{0}$ is the peak temperature in the reaction zone. Since the chemical reactions take place with finite rates, $T^{0}<T_{\text {st }}$. The reaction zone is presumed to be made up of two layers [9]. These layers are the oxidation layer of thickness of the order of $\epsilon$, and an inner layer of thickness of the order of $\delta$. The ordering $\delta \ll \epsilon \ll 1$, is presumed to hold $[9,12]$. Figure 1 shows the relative location of these layers with respect to each other in terms of the stretched coordinate $\eta$, which is the independent variable in the analysis of the oxidation layer. 


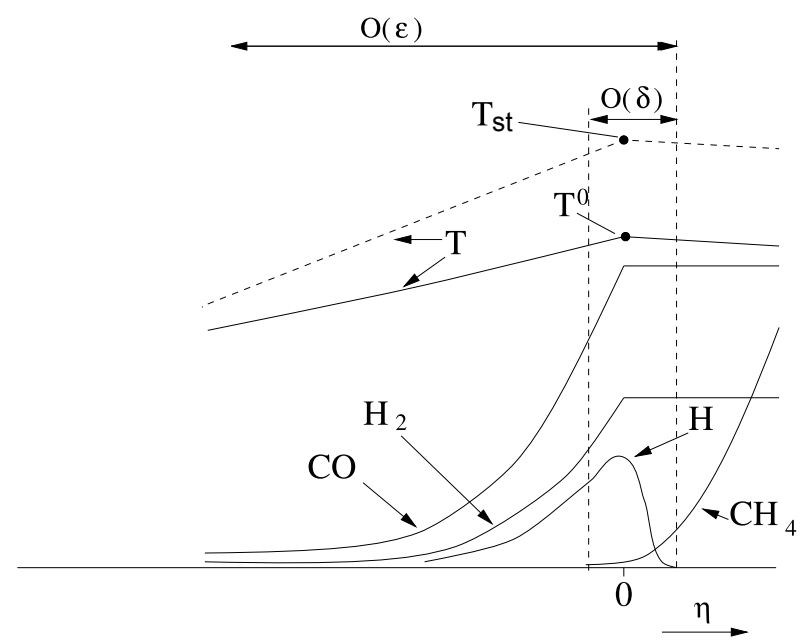

Figure 1: Schematic illustration of the structure of the reaction zone at $\xi_{\mathrm{st}}$. The figure shows the inner layer and the oxidation layer.

The stretched coordinate is so defined that the inner layer is located around $\eta=0$. The temperature and the scalar dissipation rate evaluated at $\eta=0$ are represented by $T^{0}$, and $\chi^{0}$, respectively. Superscript 0 indicates values of quantities evaluated at the inner layer. In the inner layer, the global steps I and IV are active. Fuel, $\mathrm{CH}_{4}$, is completely consumed there, and the intermediate species $\mathrm{H}_{2}$ and $\mathrm{CO}$ are formed. In the oxidation layer, the global steps, II, III, and IV, take place. Here, $\mathrm{CO}$ and $\mathrm{H}_{2}$ produced in the inner layer are oxidized to $\mathrm{CO}_{2}$ and $\mathrm{H}_{2} \mathrm{O}$. Oxygen is consumed primarily in the oxidation layer. As a consequence, in the reaction zone $X_{\mathrm{O}_{2}}, X_{\mathrm{H}_{2}}$, and $X_{\mathrm{CO}}$ are of the order of $\epsilon$. Asymptotic analysis of the oxidation layer gives values of these quantities at the inner layer. They are required for characterizing the structure of the inner layer and for calculating the scalar dissipation rate, $\chi^{0}$, at extinction. In the analysis, all rate constants and equilibrium constants 
are evaluated at $T^{0}$, and changes in the values of these rate parameters with changes in temperature are neglected [6]. The value of $T^{0}$ is also obtained in the analysis.

\subsubsection{The Oxidation Layer}

In the oxidation layer the concentration of fuel is negligible and $\mathrm{H}$ is presumed to maintain steady-state. These approximations reduce the fourstep mechanism to a two-step mechanism, (II) $\mathrm{CO}+\mathrm{H}_{2} \mathrm{O} \rightleftharpoons \mathrm{CO}_{2}+\mathrm{H}_{2}$, and (III) $\mathrm{O}_{2}+2 \mathrm{H}_{2} \rightleftharpoons 2 \mathrm{H}_{2} \mathrm{O}$. The expansions $\xi-\xi_{\mathrm{st}}=\epsilon\left(\eta+\eta^{0}\right), x_{\mathrm{CO}}=\epsilon 2 g z_{\mathrm{CO}}$, $x_{\mathrm{O}_{2}}=\epsilon g\left[z_{\mathrm{O}_{2}}-2\left(\eta+\eta^{0}\right)\right]$, are introduced where $\epsilon$ is small and the variables $\eta, z_{\mathrm{O}_{2}}$, and $z_{\mathrm{CO}}$, are presumed to be of the order of unity. From coupling relations it follows that $x_{\mathrm{H}_{2}}=\epsilon 2 g\left(z_{\mathrm{O}_{2}}-z_{\mathrm{CO}}\right)$, and from energy conservation, $\tau=\tau_{\mathrm{st}}-\epsilon\left[2 g q_{C O} z_{\mathrm{CO}}+g q_{\mathrm{O}_{2}} z_{\mathrm{O}_{2}}-s\left(\eta+\eta^{0}\right)\right][9]$. The quantity $q_{O_{2}}$ represents the fractional heat release in the steps III and IV and $q_{C O}$ the fractional heat release in II. The small expansion parameter $\epsilon$ is so chosen that it can be calculated from the equation $\epsilon=D_{\text {III }}^{-1 / 4}$, where $D_{\text {III }}$ is the Damköhler number of the global step III and is given by

$$
D_{\mathrm{III}}=2^{5 / 2} \rho^{0} g^{2} k_{5}^{0} C_{\mathrm{M}}^{0}\left(K_{1}^{0} K_{2}^{0} K_{3}^{02}\right)^{1 / 2} L e_{\mathrm{H}_{2}}{ }^{3 / 2} L e_{\mathrm{O}_{2}}{ }^{3 / 2} /\left(\chi^{0} X_{\mathrm{H}_{2} \mathrm{O}, \mathrm{st}} W_{\mathrm{N}_{2}}\right) .
$$

It has been shown in [9] that the structure of the oxidation layer can be constructed from numerical integration of two-coupled second order differential equations, one for $\mathrm{O}_{2}$ and and another for $\mathrm{CO}$. The boundary conditions for these equations are obtained from matching to the inert outer structure for large negative values of $\eta$ and to the inner layer at $\eta=0$. Figure 2 shows profiles of $z_{\mathrm{CO}}$, and $z_{\mathrm{O}_{2}}$, as functions of $\eta$. These profiles are used to obtain values of these quantities at $\eta=0$ given by $z_{\mathrm{O}_{2}}^{0}$ and $z_{\mathrm{CO}}^{0}$. They are then used 


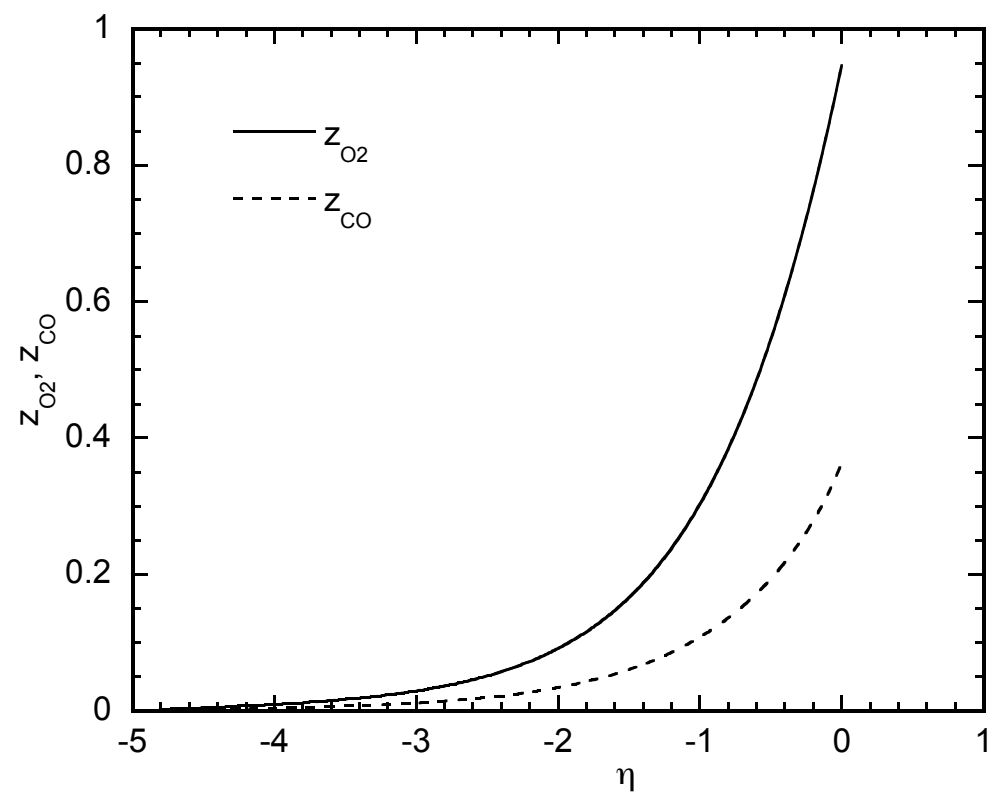

Figure 2: Profiles of $z_{\mathrm{CO}}$, and $z_{\mathrm{O}_{2}}$ as functions of $\eta$ obtained from numerical integration of equations describing the structure of the oxidation layer for $\xi_{\mathrm{st}}=0.7$ and $\chi^{0}=0.9 \mathrm{~s}^{-1}$.

to evaluate

$$
X_{\mathrm{O}_{2}}^{0}=\epsilon g L e_{\mathrm{O}_{2}}\left[z_{\mathrm{O}_{2}}^{0}-2 \eta^{0}\right], \quad X_{\mathrm{H}_{2}}^{0}=\epsilon 2 g L e_{\mathrm{H}_{2}}\left(z_{\mathrm{O}_{2}}^{0}-z_{\mathrm{CO}}^{0}\right),
$$

and the temperature $T^{0}$

$$
T^{0}=T_{\mathrm{st}}-\epsilon\left(2 g q_{C O} z_{\mathrm{CO}}^{0}+g q_{\mathrm{O}_{2}} z_{\mathrm{O}_{2}}^{0}-s \eta^{0}\right) Q_{\mathrm{F}} /\left(c_{\mathrm{p}} W_{\mathrm{N}_{2}}\right) .
$$

These quantities are required to characterize the structure of the inner layer and calculate the scalar dissipation rate at extinction. 


\subsubsection{The Structure of the Inner Layer}

The analysis of the inner layer is identical to those in $[9,12]$. The value of $\delta$ is

$$
\delta^{2}=\frac{2^{3 / 2} g^{2} k_{5}^{0} C_{\mathrm{M}}^{0} k_{8}^{0} K_{2}^{0^{1 / 2}} L e_{\mathrm{O}_{2}}{ }^{3 / 2} L e_{\mathrm{H}_{2}}{ }^{3 / 2} \epsilon^{4}}{k_{7 \mathrm{f}}^{0} k_{9}^{0} K_{1}^{0^{1 / 2}} L e_{\mathrm{F}} X_{\mathrm{O}_{2}}^{0} X_{\mathrm{H}_{2}}^{0}} .
$$

Following the analysis in [12], two coupled second-order differential equations, one for $\mathrm{CH}_{4}$ and the other for $\mathrm{H}$, are obtained. These equations include an eigenvalue $\omega$ given by

$$
\omega^{2}=\frac{2^{7 / 2} g^{4} \epsilon^{4} k_{5}^{0} C_{\mathrm{M}}^{0} k_{8}^{03} K_{2}{ }^{1 / 2} X_{\mathrm{H}_{2} \mathrm{O}, \mathrm{st}^{2}} L e_{\mathrm{H}}{ }^{2} L e_{\mathrm{O}_{2}}{ }^{3 / 2} L e_{\mathrm{H}_{2}}{ }^{3 / 2}}{k_{7 \mathrm{f}}^{0} k_{9}^{03} K_{1}^{05 / 2} K_{3}^{0} X_{\mathrm{O}_{2}}{ }^{3} X_{\mathrm{H}_{2}}^{0}{ }^{3} L e_{\mathrm{F}}} .
$$

The coupled differential equations for $\mathrm{CH}_{4}$ and $\mathrm{H}$ are required to satisfy five boundary conditions [12]. The equations are integrated numerically, with the additional boundary condition being used to obtain $\omega$.

\section{Scalar Dissipation Rate}

For given values of temperature and mass fractions of the reactants at the boundaries, the outer structure can be constructed. A value of $\eta^{0}$ is selected. The goal is to predict the scalar dissipation rate, $\chi^{0}$, that is consistent with this selected value of $\eta^{0}$. An iterative procedure is employed. It begins by selecting an appropriate value of $T^{0}$. The equations describing the structure

of the oxidation layer are integrated, and the results are used to obtain $X_{\mathrm{O}_{2}}^{0}$ and $X_{\mathrm{H}_{2}}^{0}$. The coupled differential equations for $\mathrm{CH}_{4}$, and for $\mathrm{H}$ describing the structure of the inner layer, are integrated, and the value of $\omega$ is obtained. This is compared with the value evaluated from Eq. (8). If they are not the same, the procedure is repeated with a different value of $T^{0}$ until the value of $\omega$, obtained from integration of the coupled differential equations for $\mathrm{CH}_{4}$ 
and $\mathrm{H}$, agrees with that calculated using Eq. (8). The value of $\chi^{0}$, that is consistent with the selected value of $\eta^{0}$, is calculated by recasting Eq. (4) as

$$
\chi^{0}=2^{5 / 2} \rho^{0} g^{2} k_{5}^{0} C_{\mathrm{M}}^{0}\left(K_{1}^{0} K_{2}^{0} K_{3}^{02}\right)^{1 / 2} L e_{\mathrm{H}_{2}}{ }^{3 / 2} L e_{\mathrm{O}_{2}}{ }^{3 / 2} \epsilon^{4} /\left(X_{\mathrm{H}_{2} \mathrm{O}, \mathrm{st}} W_{\mathrm{N}_{2}}\right) .
$$

The entire procedure is repeated with another selected value of $\eta^{0}$.

The classical C-shaped curve is obtained when $T^{0}$ is plotted as a function

of $\left(\chi^{0}\right)^{-1}$. The value of $\left(\chi^{0}\right)^{-1}$, where its derivative with respect to $T^{0}$ in the $\mathrm{C}$-shaped curve is zero, represents the critical condition at extinction. At this critical condition, the value of $\chi^{0}$ is denoted by $\chi_{\mathrm{q}}^{0}$. To facilitate comparison of predictions of asymptotic analysis of critical conditions of extinction, with experimental results, the scalar dissipation rate at $\xi_{\text {st }}$, denoted $\chi_{\text {st }}$, is calculated using the expression $[2,10,11]$

$$
\chi_{\mathrm{st}}=\chi^{0} \exp \left[-2 \epsilon \eta^{0} / \xi_{\mathrm{st}}\right]
$$

The value of $\chi_{\mathrm{st}}$ at extinction is represented by $\chi_{\mathrm{st}, \mathrm{q}}$.

\section{Experimental Measurements}

To test the predictions of the RRA analysis, critical conditions of extinction are measured. Steady, axisymmetric, laminar flow of two counterflowing streams toward a stagnation plane is considered. The counterflow burner used in the experimental study has two ducts. From one duct, called the fuelduct, a fuel stream made up of methane and nitrogen is injected with a speed of $V_{1}$ toward the mixing layer. From the other duct, called the oxidizer duct, an oxidizer stream made up of a mixture of oxygen and nitrogen is injected 
at a speed of $V_{2}$. The notation introduced in the RRA analysis is employed to represent the mass fractions of the reactants and their temperatures at the injection planes. The experiments are conducted with $T_{1}=T_{2}=298 \mathrm{~K}$. Hence $T_{\mathrm{u}}=298 \mathrm{~K}$, and $Q_{\mathrm{F}}=803000 \mathrm{~J}$ per mole of methane consumed [17]. Experimental data on critical conditions of extinction are obtained with the values of $Y_{\mathrm{F}, 1}$ and $Y_{\mathrm{O}_{2}, 2}$ so chosen that $T_{\mathrm{st}}=2000 \mathrm{~K}$. At these conditions, $c_{\mathrm{p}, \mathrm{st}}=1300 \mathrm{~J} /(\mathrm{kg} \cdot \mathrm{K})[17]$. It follows from Eq. (2) that $Y_{\mathrm{F}, 1} \xi_{\mathrm{st}}=0.044$ and from Eq. (1) that $Y_{\mathrm{O}_{2}, 2}\left(1-\xi_{\mathrm{st}}\right)=0.177$. These relations are used to evaluate $Y_{\mathrm{F}, 1}$ and $Y_{\mathrm{O}_{2}, 2}$ from selected values of $\xi_{\mathrm{st}}$. The experiments are conducted for values of $0.044 \leq \xi_{\mathrm{st}}<0.823$. For, $\xi_{\mathrm{st}}=0.044, Y_{\mathrm{F}, 1}=1$, and for $\xi_{\mathrm{st}}=0.823$, $Y_{\mathrm{O}_{2}, 2}=1$.

In experimental studies, a convenient parameter to characterize the residence time is the reciprocal of the strain rate. For the counterflow configuration considered here, the value of the strain rate, defined as the normal gradient of the normal component of the flow velocity, changes from the exit of the fuel duct to the exit of the oxidizer duct [18]. It is discontinuous across the stagnation plane. The local strain rate on the oxidizer side of the stagnation plane, is $a_{2}=\left(2\left|V_{2}\right| / L\right)\left[1+\left|V_{1}\right| \sqrt{\rho_{1}} /\left(\left|V_{2}\right| \sqrt{\rho_{2}}\right)\right][18]$. Here, $\rho_{1}$ and $\rho_{2}$ are the densities of the reactant streams at the injection plane of the fuel duct and at the injection plane of the oxidizer duct, respectively. The separation distance between the ducts is $L$. The quantity $a_{2}$ is a good measure of the characteristic residence time for $0<\xi_{\text {st }} \leq 0.5$. On the fuel side of the stagnation plane, the local strain rate $a_{1}=a_{2} \sqrt{\rho_{2} / \rho_{1}}$, and it represents a better measure of the characteristic residence time for $0.5 \leq \xi_{\mathrm{st}}<1.0$. At 
$\xi_{\mathrm{st}}=0.5$, the strain rates of these two measures are discontinuous but do not differ much. The expressions for the strain rates are an exact solution of the inviscid, but rotational, equation of motion obtained in the asymptotic limit of large Reynolds numbers of the reactant streams at the injection planes [18] and are, therefore, fundamentally appropriate representations of the residence time [19].

At a selected $\xi_{\text {st }}$, the values $Y_{\mathrm{F}, 1}$ and $Y_{\mathrm{O}_{2}, 2}$ are calculated and a flame is established at some value of the strain rate less than the extinction strain rate. The strain rate is then increased by increasing $V_{1}$ and $V_{2}$ simultaneously until extinction is observed. The strain rate at extinction, $a_{2, \mathrm{q}}$ and $a_{1, \mathrm{q}}$, are recorded as a function of $\xi_{\mathrm{st}}$. The quantities $a_{2, \mathrm{q}}$ and $a_{1, \mathrm{q}}$ are the values of $a_{2}$ and $a_{1}$ at extinction. To facilitate comparison of experimental measurements with predictions of RRA analysis, the scalar dissipation rate at extinction, $\chi_{\mathrm{st}, \mathrm{q}}$ is calculated from $a_{\mathrm{q}}$ using a relation derived by Kim and Williams [20]. This relation is

$$
\chi_{\mathrm{st}, \mathrm{q}}=\frac{a_{2, \mathrm{q}}}{2 \pi} \frac{3\left[\left(T_{\mathrm{st}} / T_{\mathrm{u}}\right)^{1 / 2}+1\right]^{2}}{2\left(T_{\mathrm{st}} / T_{\mathrm{u}}\right)^{1 / 2}+1} \times \exp \left\{-2\left[\operatorname{erfc}^{-1}\left(2 \xi_{\mathrm{st}}\right)\right]^{2}\right\} .
$$

Here $\mathrm{erfc}^{-1}$ is the inverse of the complimentary error function. Equation (11) is used for evaluating $\chi_{\mathrm{st}, \mathrm{q}}$ from experimental data for $\xi_{\mathrm{st}}<0.5$. For $\xi_{\mathrm{st}}>0.5$, $\chi_{\text {st,q }}$ is calculated with $a_{2, \mathrm{q}}$ and $\xi_{\text {st }}$ in Eq. (11), respectively, replaced by $a_{1, \mathrm{q}}$ and $\left(1-\xi_{\mathrm{st}}\right)$.

Figure 3 shows the measured strain rates at extinction, and the scalar dissipation rate at extinction $\chi_{\mathrm{st}, \mathrm{q}}$ as functions of $\xi_{\mathrm{st}}$ calculated there from 


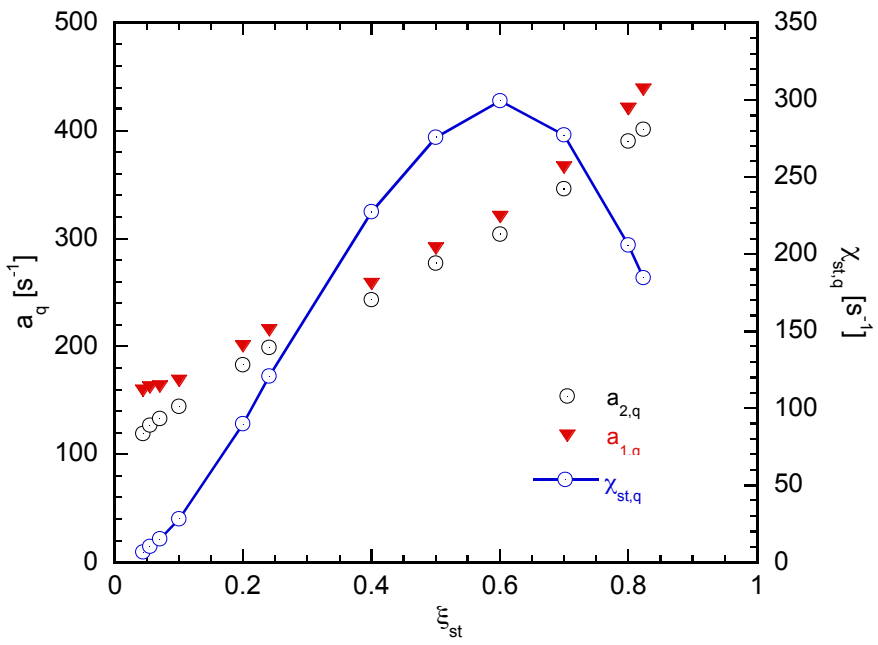

Figure 3: The measured local strain rates at extinction, $a_{2, \mathrm{q}}$ and $a_{1, \mathrm{q}}$, and the scalar dissipation rate at extinction $\chi_{\mathrm{st}, \mathrm{q}}$ calculated using Eq. (11) as functions of $\xi_{\mathrm{st}}$ at fixed values of $T_{\mathrm{st}}=2000 \mathrm{~K}$.

by Eq. (11). In general, at a given $\xi_{\mathrm{st}}$, the values of $a_{2, \mathrm{q}}$ and $a_{1, \mathrm{q}}$ are nearly the same. The experimental data shows that the strain rates at extinction increase with increasing $\xi_{\text {st }}$. These results are in agreement with previous findings [14]. It is noteworthy that, as $\xi_{\text {st }}$ increases from small values, the scalar dissipation rate at extinction, $\chi_{\mathrm{st}, \mathrm{q}}$, first increases and then decreases. Computations were performed using the San Diego Mechanism [21], and the flame structure and critical conditions of extinction were predicted. The predictions were quite similar to those obtained using the USC II mechanism [22]. The predictions showed that the strain rate at extinction increased with increasing $\xi_{\text {st }}$, while the scalar dissipation rate at extinction first increased and then decreased, in agreement with the trend obtained in the measurements. The computational predictions of the strain rate at extinction were 
close to the measurements for small values of $\xi_{\mathrm{st}}$, while for larger values of $\xi_{\mathrm{st}}$, the predictions were higher than the measurements by a factor not greater than 1.5.

To obtain an improved understanding for the reasons for the increase in values of $a_{2, \mathrm{q}}$ and $a_{1, \mathrm{q}}$ with increase in $\xi_{\mathrm{st}}$, the temperature profile and profiles of mass fraction of various species were calculated using detailed chemistry at a fixed value of the strain rate and various values of $\xi_{\text {st }}$. For all values

of $\xi_{\text {st }}$ there was nearly complete consumption of $\mathrm{CH}_{4}$, but there was leakage of $\mathrm{O}_{2}$ from the reaction zone (measured as the value of the mass fraction of $\mathrm{O}_{2}$ at $\xi_{\text {st }}$ ). Net rates of consumption of $\mathrm{CH}_{4}$ and $\mathrm{O}_{2}$ were calculated. With increasing $\xi_{\text {st }}$, the thickness of the regions where these reactants are consumed are found to increase, and leakage of oxygen at conditions close to extinction was found to increase.

\section{Results and Discussion}

To evaluate critical conditions of extinction, for simplicity, the concentration of the third body, $C_{\mathrm{M}}^{0}$, the density, $\rho^{0}$, and the heat capacity, $c_{\mathrm{p}}$, are evaluated using the relations $C_{\mathrm{M}}^{0}=20270 / T^{0} \mathrm{~mol} / \mathrm{m}^{3}, \rho^{0}=351 / T^{0} \mathrm{~kg} / \mathrm{m}^{3}$, and $c_{\mathrm{p}}=1045+0.24 T-0.2 T^{-2} \mathrm{~J} /(\mathrm{kg} \cdot \mathrm{K})[9,12,23]$. The molecular weights are $W_{\mathrm{F}}=0.016 \mathrm{~kg} / \mathrm{mol}, W_{\mathrm{N}_{2}}=0.028 \mathrm{~kg} / \mathrm{mol}, W_{\mathrm{O}_{2}}=0.032 \mathrm{~kg} / \mathrm{mol}$, and $W_{\mathrm{H}_{2}}=0.002 \mathrm{~kg} / \mathrm{mol}$, and the Lewis numbers of various species are $L e_{\mathrm{F}}=$ 0.97, $L e_{\mathrm{H}_{2}}=0.3, L e_{\mathrm{CO}}=1.11$, and $L e_{\mathrm{H}}=0.2$ [23], and the fractional heat release $q_{O_{2}}=0.602$, and $q_{C O}=0.052$. 


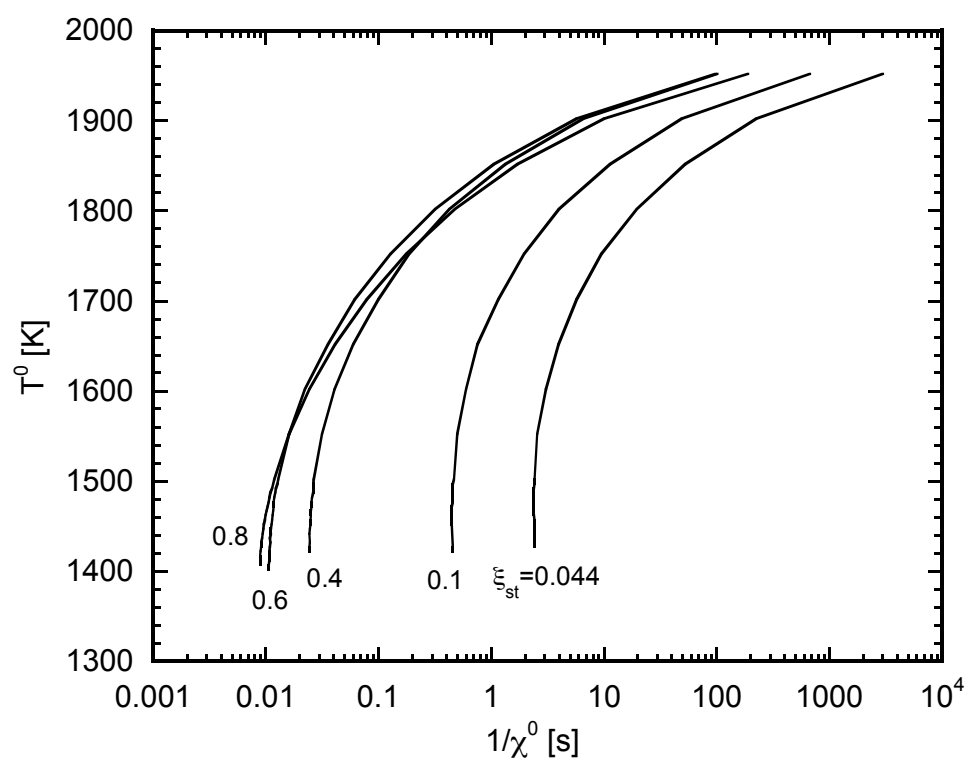

Figure 4: The flame temperature, $T^{0}$, as a function of $\left(\chi^{0}\right)^{-1}[\mathrm{~s}]$, for various values of $\xi_{\mathrm{st}}$ and at fixed $T_{\mathrm{st}}=2000$.

Figure 4 shows $T^{0}$ and Fig. 5 shows $X_{\mathrm{O}_{2}}^{0}$ both as functions of $\left(\chi^{0}\right)^{-1}$ for various values of $\xi_{\text {st }}$. All show the classical C-shaped behavior. The upper branches of the C-shaped curves for $T^{0}$ vs. $\left(\chi^{0}\right)^{-1}$ and the lower branches for $X_{\mathrm{O}_{2}}^{0}$ vs. $\left(\chi^{0}\right)^{-1}$ are stable. With decreasing values of $\left(\chi^{0}\right)^{-1}$, the flame temperature decreases and the oxidizer leakage represented by $X_{\mathrm{O}_{2}}^{0}$ increases. All curves show that there are no solutions for values of $\left(\chi^{0}\right)^{-1}$ below a critical value. This critical value of $\chi^{0}$ is the scalar dissipation rate at extinction represented by $\chi_{\mathbf{q}}^{0}$. It is noteworthy, that at extinction the values of $T^{0}$ are nearly the same, while the value of $X_{\mathrm{O}_{2}}^{0}$ increases with increasing $\xi_{\mathrm{st}}$. The value of the scalar dissipation rate at extinction evaluated at $\xi_{\mathrm{st}}, \chi_{\mathrm{st}, \mathrm{q}}$, is obtained from $\chi_{q}^{0}$ using Eq. (10). 


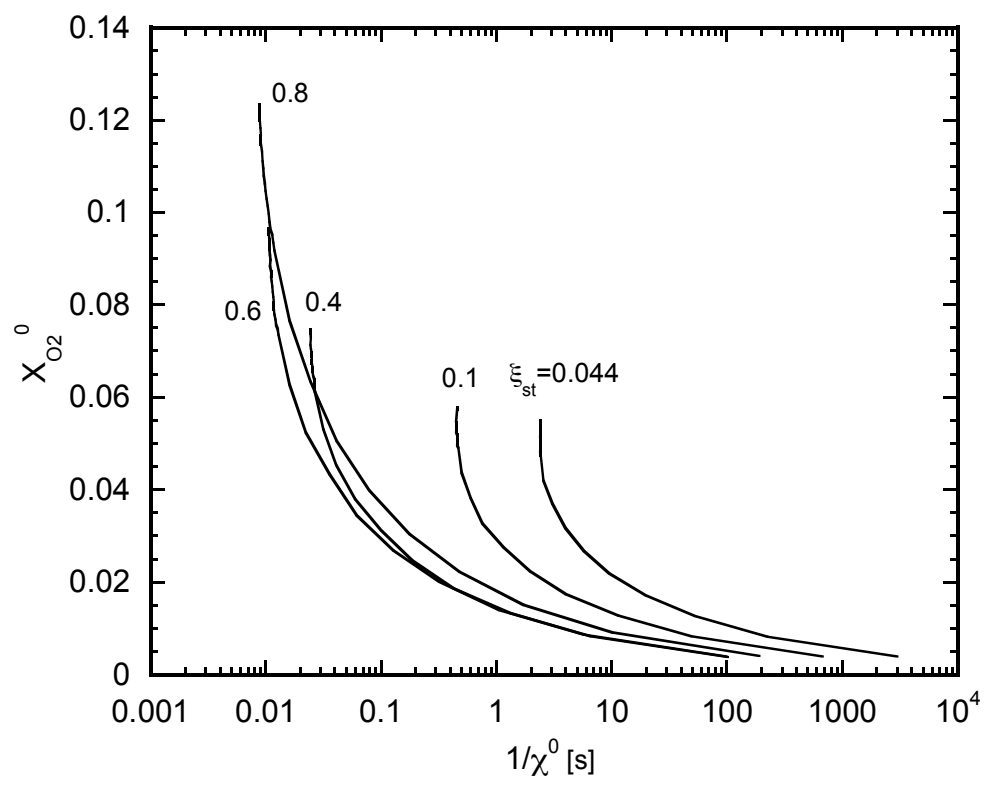

Figure 5: The normalized mass fraction of $\mathrm{O}_{2}, X_{\mathrm{O}_{2}}^{0}$, as a function of $\left(\chi^{0}\right)^{-1}[\mathrm{~s}]$, for various values of $\xi_{\text {st }}$ and at fixed $T_{\text {st }}=2000$.

Fig. 6 shows the ratio $\chi_{\mathrm{st}, \mathrm{q}} / \chi_{\mathrm{st}, \mathrm{q}, \mathrm{ref}}$ as a function of $\xi_{\mathrm{st}}$. The symbols are experimental data and the solid lines are predictions of the asymptotic analysis. The quantity $\chi_{\mathrm{st}, \mathrm{q}, \mathrm{ref}}$ is the maximum value of $\chi_{\mathrm{st}, \mathrm{q}}$. In the asymptotic analysis, this peak value of $127 \mathrm{~s}^{-1}$ is attained at $\xi_{\mathrm{st}}=0.7$. The symbols in Fig. 6 represent values of $\chi_{\mathrm{st}, \mathrm{q}}$ calculated using Eq. (11) and the experimental data for $a_{2, \mathrm{q}}$ for $0<\xi_{\mathrm{st}} \leq 0.5$ and $a_{1, \mathrm{q}}$ for $0.5<\xi_{\mathrm{st}}<1$. The predictions agree well with experimental data. The predictions show that $\chi_{\mathrm{st}, \mathrm{q}}$ first increases with increasing $\xi_{\text {st }}$, reaches a peak value and then decreases. Fig. 7 shows values of $z_{\mathrm{O}_{2}}^{0}, z_{\mathrm{CO}}^{0}$, and $\eta^{0}$ as functions of $\xi_{\text {st }}$. The changes in values of these quantities with changes in $\xi_{\text {st }}$ are small. 


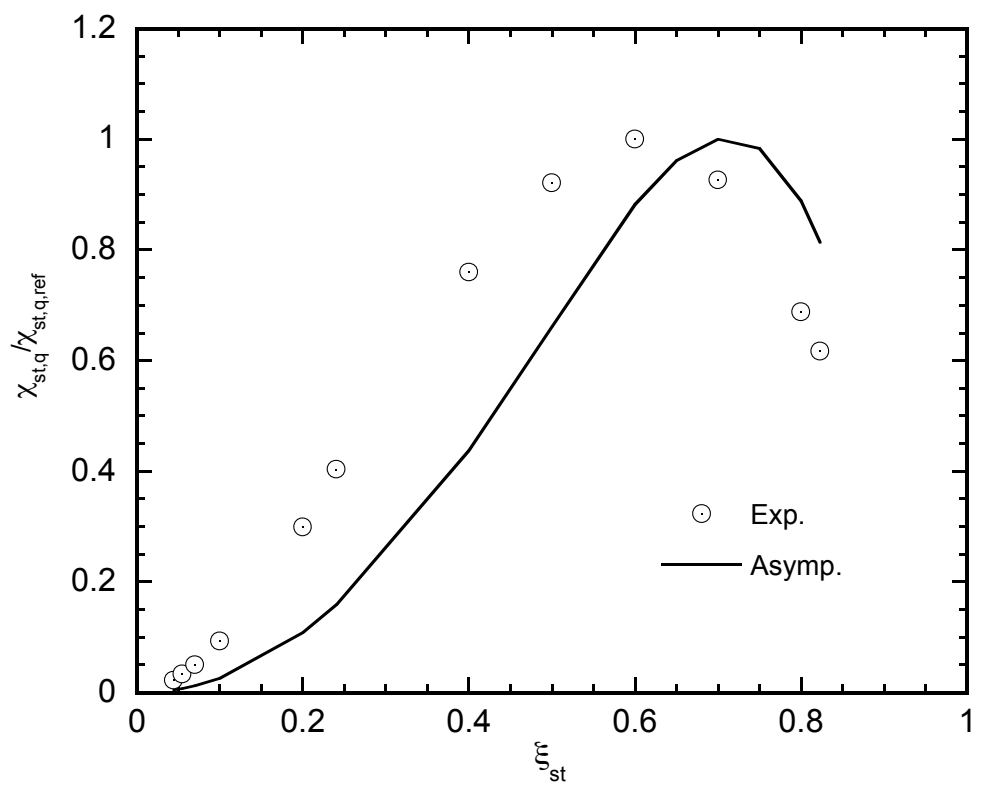

Figure 6: The ratio $\chi_{\mathrm{st}, \mathrm{q}} / \chi_{\mathrm{st}, \mathrm{q}, \mathrm{ref}}$ at extinction for various values of $\xi_{\mathrm{st}}$ and at fixed $T_{\mathrm{st}}=$ 2000. The symbols represent experimental data and the solid lines are predictions of the asymptotic analysis.

\section{The Influence of $\xi_{\text {st }}$ On Extinction}

The results of the asymptotic analysis are used to obtain an improved understanding of the influence of $\xi_{\text {st }}$ on critical conditions of extinction. Figure 8 shows values of $\epsilon$ and $\delta$ as a function of $\xi_{\mathrm{st}}$. For all $\xi_{\mathrm{st}}$, the value of $\delta$ is less than that of $\epsilon$, and both are less than 0.1. This confirms the ordering $\delta \ll \epsilon \ll 1$ employed in the analysis. A key finding is that the value of $\epsilon$ and $\delta$ increases with $\xi_{\text {st }}$ and is consistent with computational predictions of changes in thickness of the regions of oxygen consumption and fuel consumption.

Equation (9) shows that $\chi^{0}$ depends on $T^{0}, \epsilon, g$ and $X_{\mathrm{H}_{2} \mathrm{O}, \mathrm{st}}$, and Eq. 


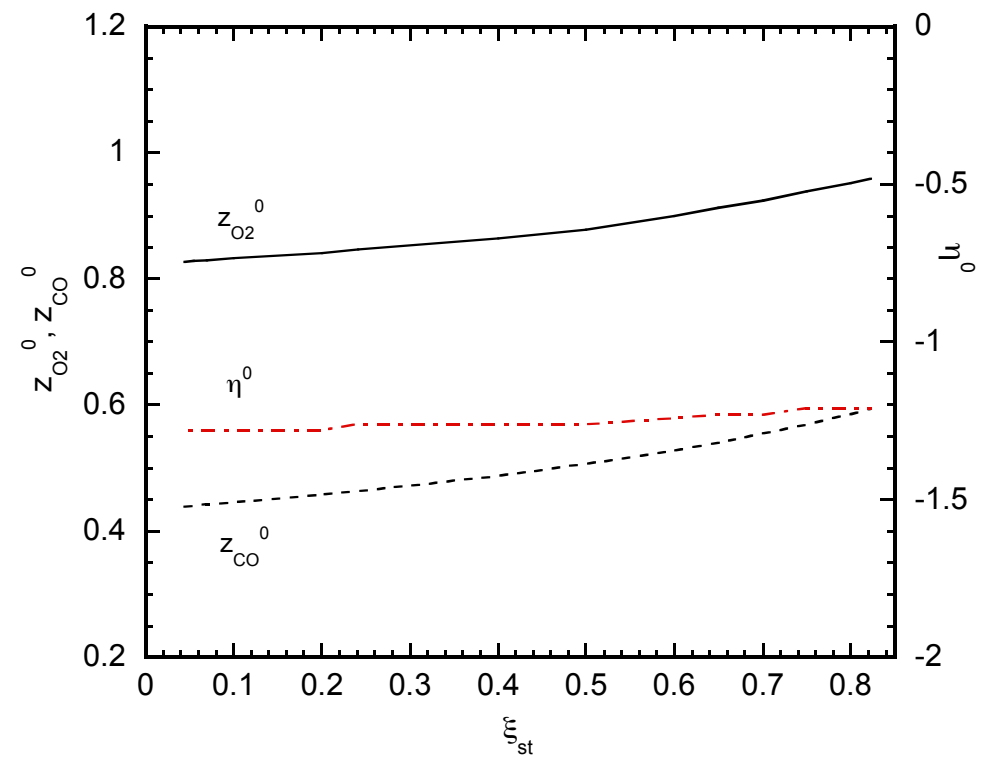

Figure 7: Values of $z_{\mathrm{O}_{2}}^{0}, z_{\mathrm{CO}}^{0}$, and $\eta^{0}$ as function of $\xi_{\mathrm{st}}$.

(6) shows that $\epsilon$ depends on $g, z_{\mathrm{O}_{2}}^{0}, z_{\mathrm{CO}}^{0}$, and $\eta^{0}$. At fixed $T_{\mathrm{st}}=2000 \mathrm{~K}$ considered here, $\tau_{\text {st }}=0.0772, g=0.0772 /\left[\xi_{\text {st }}\left(1-\xi_{\text {st }}\right)\right], X_{\mathrm{H}_{2} \mathrm{O}, \mathrm{st}}=0.1552$, and $s / g \approx\left(1-\xi_{\mathrm{st}}\right)$. Results of the asymptotic analysis described here shows that at conditions close to extinction, changes in the values of $T^{0}, z_{\mathrm{O}_{2}}^{0}, z_{\mathrm{CO}}^{0}$, and $\eta^{0}$ with changes in $\xi_{\text {st }}$ are small, and their values are, respectively, $1400 \mathrm{~K}$, $0.9,0.4,-1.2$. After substitution of these values in Eqs. (6) and (5) it follows that

$$
\epsilon \approx 0.33\left[\xi_{\mathrm{st}}\left(1-\xi_{\mathrm{st}}\right)\right] /\left(2.0-1.3 \xi_{\mathrm{st}}\right), \quad X_{\mathrm{O}_{2}}^{0}=0.09 /\left(2.0-1.3 \xi_{\mathrm{st}}\right)
$$

The peak value of $\epsilon$ is around $\xi_{\text {st }}=0.63$. The value of $\epsilon$ obtained from Eq. (12) is plotted in Fig. 8, and it is close to the values obtained from the asymptotic analysis. Equation (12) shows that the leakage of oxygen from the reaction zone increases with increasing $\xi_{\text {st }}$. This is in agreement with the 


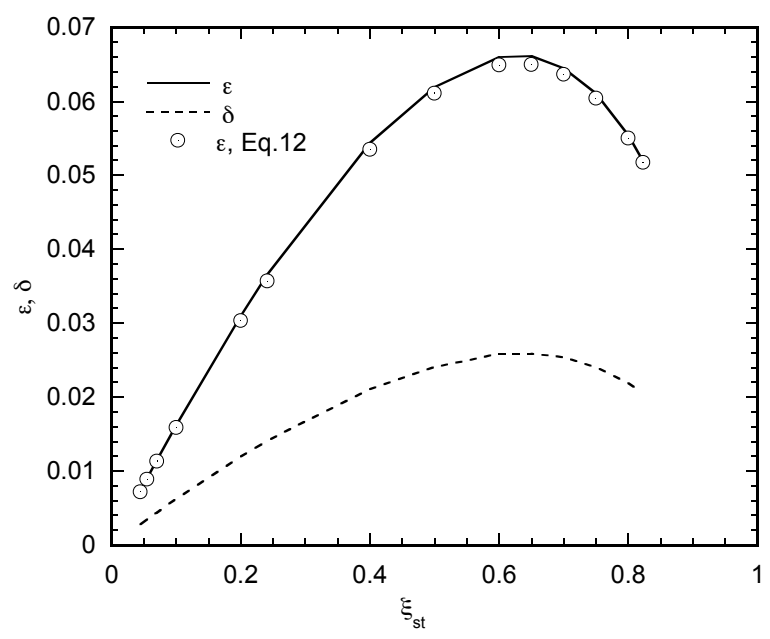

Figure 8: Predicted value of $\epsilon$ and $\delta$ as a function of $\xi_{\mathrm{st}}$. The figure also shows the approximate value of $\epsilon$ calculated using Eq. (12)

results shown in Fig. 5 and with predictions using detailed chemistry. Equation (7) shows that $\delta$ is proportional to $\epsilon$. Thus, changes in the thickness of the inner layer are similar to that of the oxidation layer.

It follows from Eq. (12) that at fixed $T_{\mathrm{st}}=2000 \mathrm{~K}$ and close to critical conditions of extinction, $\epsilon g \approx 0.025 /\left(2.0-1.3 \xi_{\text {st }}\right)$. Hence the scalar dissipation rate at extinction, $\chi_{\mathrm{q}}^{0}$, deduced from Eq. (9) is

$$
\chi_{\mathrm{q}}^{0} \propto \epsilon^{2} /\left(2.0-1.3 \xi_{\mathrm{st}}\right)^{2},
$$

Equation (13) shows that $\chi_{\mathrm{q}}^{0}$ is proportional to $\epsilon^{2}$. For fixed $\left(T_{\mathrm{st}}-T_{\mathrm{u}}\right)$, the quantity $\xi_{\text {st }}\left(1-\xi_{\text {st }}\right)$ is inversely proportional to the sum of the absolute values of the temperature gradients in the outer structure. Therefore it follows from Eqs. (12) and (13) that $\chi_{q}^{0}$ is inversely proportional to the square of the sum of the absolute values of the temperature gradients in the outer 
structure.

\section{Concluding Remarks}

The asymptotic analysis gives a clear description of the influence of $\xi_{\text {st }}$ on critical conditions of extinction of nonpremixed methane flames. At fixed $T_{\text {st }}$ starting from small $\xi_{\text {st }}$, with increasing $\xi_{\text {st }}$, predictions of the asymptotic analysis show that the scalar dissipation rate at extinction, $\chi_{\mathrm{st}, \mathrm{q}}$, first increases, attains a peak value, and then decreases. Experimental data confirm the accuracy of this prediction. A noteworthy observation is that with increasing $\xi_{\text {st }}$, the thicknesses of the reaction zones first increase and then decrease. The sum of the absolute values of the temperature gradients in the outer structure is inversely proportional to the thickness of the reaction zone. Thus changes in the values of $\chi_{\text {st,q }}$ with changes in $\xi_{\text {st }}$ follow the changes in values of the thickness of the oxidation layer and as a consequence the sum of the absolute value of the temperature gradient in the outer structure. Another important prediction is that with an increase in values of $\xi_{\text {st }}$, the leakage of oxygen through the reaction zone increases. This occurs because of the shallower concentration gradient on the oxygen side, which affects its evolution through the oxygen-consumption $(\epsilon)$ layer.

Computational investigation also was carried out to characterize the influence of $\xi_{\text {st }}$ on critical conditions of extinction of methane flames with the chemistry represented by a one-step irreversible reaction Fuel + Oxygen $\rightarrow$ Products. It was found that (1) for small $\xi_{\text {st }}$ there was leakage of fuel, while for small $\left(1-\xi_{\mathrm{st}}\right)$ there was leakage of oxygen, and $(2)$ there were very little 
changes in the values of the strain rate at extinction with changes in the values of $\xi_{\text {st }}$. Thus, there are fundamental differences in predictions obtained employing detailed chemistry and one-step chemistry.

\section{Acknowledgments}

The authors acknowledge the valuable assitance of Dr. Ulrich Niemann with the experimental part of this work. The authors thank Professor P. D. Ronney and Professor F. A. Williams for their comments and helpful discussions. The research at UCSD is supported by the National Science Foundation, award \# CBET-1404026.

\section{References}

[1] N. Peters, Combustion Science and Technology 30 (1983) 1-17.

[2] N. Peters, Progress in Energy and Combustion Science 10 (1984) 319339.

[3] A. Liñán, Acta Astronautica 1 (1974) 1007-1039.

[4] F. A. Williams, Combustion Theory, Addison-Wesley Publishing Company, Redwood City, CA, 2 edition, 1985.

[5] A. Liñán, F. A. Williams, volume 34 of Oxford Engineering Science Series, Oxford University Press, New York, 1993.

[6] K. Seshadri, Proceedings of the Combustion Institute 26 (1996) 831-846.

[7] K. Seshadri, C. Trevino, Combustion Science and Technology 64 (1989) 243-261. 
[8] U. Niemann, K. Seshadri, F. A. Wiliams, Combustion Theory and Modelling 17 (2013) 563-576.

[9] K. Seshadri, X. S. Bai, Combustion Science and Technology 187 (2015) $3-26$.

[10] K. Seshadri, N. Peters, Combustion and Flame 73 (1988) 23-44.

[11] B. Yang, K. Seshadri, Combustion Science and Technology 88 (1992) $115-132$.

[12] X. S. Bai, K. Seshadri, Combustion Theory and Modelling 3 (1999) $51-75$.

[13] A. Grudno, K. Seshadri, Combustion Science and Technology, 112 (1996) 199-210.

[14] R. Chen, R. L. Axelbaum, Combustion and Flame 142 (2005) 62-71.

[15] H. Song, P. Wang, R. S. Boles, D. Matinyan, H. Prahanphap, J. Piotrowicz, P. D. Ronney, in: Paper \# 1B05 presented at the 2016 Spring Technical Meeting of the Western States Section Combustion Institute, University of Washington, Seattle, WA.

[16] N. Peters, in: N. Peters, B. Rogg (Eds.), Reduced Kinetic Mechanisms for Applications in Combustion Systems, volume m15 of Lecture Notes in Physics, Springer-Verlag, Heidelberg, 1993, pp. 1-13.

[17] NIST-JANAF Thermochemical Tables, http://kinetics.nist.gov/janaf/, 2013. 
[18] K. Seshadri, F. A. Williams, International Journal of Heat and Mass Transfer 21 (1978) 251-253.

[19] H. Schlichting, Boundary-Layer Theory, Mc Graw Hill, Inc, 1968.

[20] J. S. Kim, F. A. Williams, SIAM Journal on Applied Mathematics 53 (1993) 1551-1566.

[21] P. Saxena, Numerical and Experimental Studies of Ethanol Flames and Autoignition Theory for Higher Alkanes, Ph.d thesis, University of California at San Diego, Department of Mechanical and Aerospace Engineering, 2007.

[22] H. Wang, X. You, A. V. Joshi, S. G. Davis, A. Laskin, F. Egolfopoulos, C. K. Law, Usc mech version II. HighTemperature Combustion Reaction Model of H2/CO/C1-C4 Compounds. http://ignis.usc.edu/usc_mech_ii.htm, 2007.

[23] M. D. Smooke, V. Giovangigli, in: M. D. Smooke (Ed.), Reduced Kinetic Mechanisms and Asymptotic Approximations for Methane-Air Flames, volume 384 of Lecture Notes in Physics, Springer Verlag Berlin Heidelberg, 1991, pp. 1-28. 


\section{List of Tables}

1 Rate data. Units are moles, cubic centimeters, seconds, kJoules, Kelvin. . . . . . . . . . . . . . . . . . 7

\section{List of Figures}

1 Schematic illustration of the structure of the reaction zone at $\xi_{\text {st. }}$. The figure shows the inner layer and the oxidation layer. . 9

2 Profiles of $z_{\mathrm{CO}}$, and $z_{\mathrm{O}_{2}}$ as functions of $\eta$ obtained from numerical integration of equations describing the structure of the oxidation layer for $\xi_{\mathrm{st}}=0.7$ and $\chi^{0}=0.9 \mathrm{~s}^{-1} \ldots \ldots \ldots 11$

3 The measured local strain rates at extinction, $a_{2, \mathrm{q}}$ and $a_{1, \mathrm{q}}$, and the scalar dissipation rate at extinction $\chi_{\mathrm{st}, \mathrm{q}}$ calculated using Eq. (11) as functions of $\xi_{\text {st }}$ at fixed values of $T_{\text {st }}=2000 \mathrm{~K} .16$

4 The flame temperature, $T^{0}$, as a function of $\left(\chi^{0}\right)^{-1}[\mathrm{~s}]$, for various values of $\xi_{\text {st }}$ and at fixed $T_{\text {st }}=2000 \ldots \ldots$. . . . . 18

5 The normalized mass fraction of $\mathrm{O}_{2}, X_{\mathrm{O}_{2}}^{0}$, as a function of $\left(\chi^{0}\right)^{-1}[\mathrm{~s}]$, for various values of $\xi_{\mathrm{st}}$ and at fixed $T_{\mathrm{st}}=2000 \ldots .19$

6 The ratio $\chi_{\mathrm{st}, \mathrm{q}} / \chi_{\mathrm{st}, \mathrm{q}, \mathrm{ref}}$ at extinction for various values of $\xi_{\mathrm{st}}$ and at fixed $T_{\mathrm{st}}=2000$. The symbols represent experimental data and the solid lines are predictions of the asymptotic analysis. . . . . . . . . . . . . . . 20

$7 \quad$ Values of $z_{\mathrm{O}_{2}}^{0}, z_{\mathrm{CO}}^{0}$, and $\eta^{0}$ as function of $\xi_{\mathrm{st}}$. . . . . . . . . 21

8 Predicted value of $\epsilon$ and $\delta$ as a function of $\xi_{\text {st }}$. The figure also shows the approximate value of $\epsilon$ calculated using Eq. (12) . . 22 